commenced it is important that the delicate new skin at the growing edges should not be damaged, as it easily can be, by the application and removal of the dressing, or by the application of antiseptics. One of the best dressings at this stage is wool soaked in sterilised oil. The oil protects the delicate granulations and prevents their being damaged when the dressings are removed. If, as is sometimes the case, the wound shows a tendency to stop healing, some stimulating dressing should be applied, such as pure ichthyol, lotio rubra or Friar's balsam, but it should not be applied too frequently. When the wound is nearly healed I prefer to put no dressing into the wound, but only to apply a pad of wool over the anus, any tendency to bridging being prevented by the application of a probe if necessary. The patient should not be allowed to sit up or walk until the wound is soundly healed. It is often a great temptation, both to the medical man and the patient, to allow the latter to get about a little when the wound is nearly. healed, but this often results in the wound breaking down again or refusing to heal further. The reason for this is that the rectal veins have no valves, and consequently in the erect position there is considerable pressure, from the effect of gravity, on the column of blood in the large venous trunks; and this extra pressure, by interfering with the circulation through the tissues of the wound, delays the healing. If the fistula has been sewn up the after-treatment is the same as for any other sutured wound.

Cavendish-place, $\mathrm{w}$.

\section{GRAPHIC RECORDS OF RESPIRATORY PARALYSIS.}

By G. A. SUTHERLAND, M.D. EDIN., F.R.C.P. LOND., PHYSICIAN TO THE HAMPSTEAD AND YORTH-WEST LONDON HOSPITAL AND TO THE PADDINGTON GREEN CHILDREX'S HOSPITAL.

PARALYSIS of the respiratory muscles may not be marked by any very obvious signs, and even when suspected its presence or absence may not be easy to determine, especially in the milder forms. The two chief varieties-namely, that in which the diaphragm is paralysed and that in which the costal muscles are paralysed-may be easily recognised by means of graphic records. The instrument I have used is Mackenzie's ink polygraph. Two rubber bags attached by rubber tubing to the tambours are used. One bag, slightly inflated, is placed over the lower part of the abdomen and fixed in position with a flannel bandage. The other is placed over the chest abcut the mid-sternal region and similarly fixed.

Under normal conditions of respiration a double tracing similar to that shown in Fig. 1 will be obtained. Inspiration is indicated by the upstroke (I) of the curve, expiration

FIG. 1.
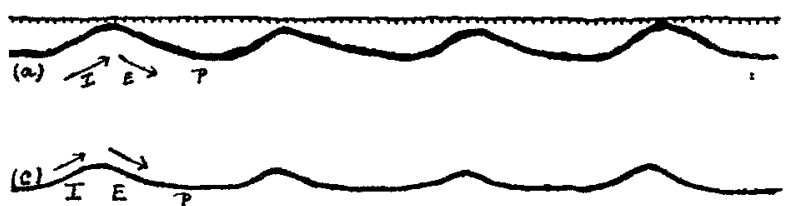

Tracing of (a) normal abdominal, and (c) costal breathing.

by the downstroke (E), and the period of pause (P) is the more or less horizontal part of the tracing. The inspiratoryexpiratory rise and fall will always be present, while the tracing of the pause period will vary greatly with the rate of the breathing and the rate at which the tracing paper is moving. As regards the character of the tracings, it will be seen that they follow similar curves, because the abdomen and the thorax expand simultaneously and diminish simultaneously. As indicated by the arrow heads in Fig. 1 the two pens are always moving in the same line of curve, every rise in the one being accompanied by a rise in the other, and so on. While the tracing is being taken one can see the two pens rising and falling on the paper at the same time, and at a glance one can tell that the respiratory movements are normal in direction. In order to gain information from watching the movements of the pens in other than normal conditions it is advisable always to use the one tambour for abdominal respiration and the other for thoracic. In the tracings given here the upper line always represents the abdominal breathing, and the lower the costal.

Fig. 2 is a tracing taken from a boy two years old who was suffering from paralysis of the diaphragm after an attack

FIG. 2.

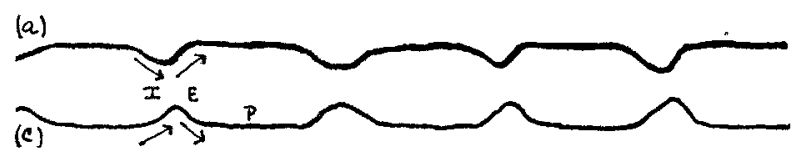

Tracing in paralysis of the diaphragm. The abdominal curve (a) is inverted; the costal (c) is normal.

of diphtheria. Some discussion had arisen as to whether the diaphragm was really paralysed or not. The tracing showed at a glance the characteristic change in the curve due to the loss of diaphragmatic action. The lower of the tracings, taken from the thorax, shows a perfectly normal condition. The upper one, taken from the abdomen, shows a series of inverted curves. Inspiration is here marked by a descent and expiration by an ascent of the curve, while the pause occupies the highest part of the tracing. As indicated by the arrow heads, the two pens during inspiration are moving towards each other, and during expiration are moving away from each other. When one watched the movements of the pens the same thing was shown even more strikingly than in the tracing, and the contrast with the movements seen in normal respiration was very marked. We find, then, a completely inverted tracing of the abdominal movement in paralysis of the diaphragm. The explanation is that in diaphragmatic paralysis the abdomen sinks in during inspiration and expands during expiration.

The result of paralysis of the intercostal muscles, as. indicated by graphic methods, is shown in Fig. 3. This

FIG. 3.

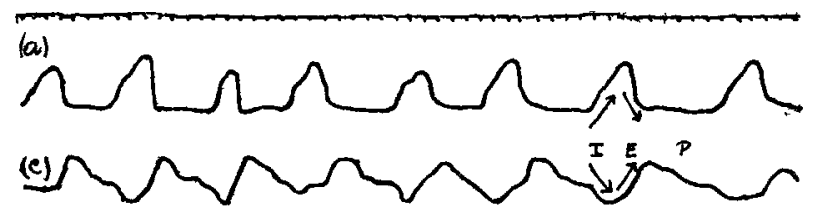

Tracing in paralysis of the costal muscles. The abdominal curve (a) is normal, the costal (c) is inverted.

tracing was taken from a case of acute ascending poliomyelitis, the patient being a girl aged 16 years. The abdominal tracing is of normal type, and there was no doubt. clinically that the diaphragm was acting well. At first sight it might appear that the costal tracing is also normal. If, however, the curves are compared with those from the abdomen it will be seen that they are completely inverted. Inspiration is marked by a downstroke and expiration by an upstroke. As indicated by the arrow-heads, the two pens during inspiration are moving away from each other, and during expiration are moving towards each other. This is. exactly the opposite movement from that associated with paralysis of the diaphragm, and is quite different from the condition in normal respiration. One often has considerable difficulty clinically in telling by means of inspection and palpation when and how far intercostal paralysis is present. A tracing such as the above gives clear and definite evidence of the costal movements and their relation to those of the abdomen. In intercostal paralysis the sterno-mastoid and scalene muscles fix the upper part of the thorax, and the diaphragmatic movement brings down and inflates the lung. This is accompanied by a falling in of the thoracic wall from atmospheric pressure, and the period is marked in the costal tracing by the downward stroke. The more sudden upstroke during expiration would seem to indicate a rebound on the part of the ribs when the diaphragmatic traction was removed and the external abdominal muscles contracted. A week after the above tracing was taken I proceeded to take another, and found that costal action, although still defective. was of the normal type, as shown by the symmetrical nature of the curves.

Wimpole-street, $\mathrm{W}$, 